\title{
First Principles Study of Water-Based Self-Assembled Nanobearing Effect in CrN/TiN Multilayer Coatings
}

\author{
David Holec ${ }^{1, a}$, Jörg Paulitsch ${ }^{2}$ and Paul H. Mayrhofer ${ }^{2, b}$
}

${ }^{1}$ Department of Physical Metallurgy and Materials Testing, Montanuniversität Leoben, Franz-JosefStrasse 18, A-8700 Leoben, Austria

${ }^{2}$ Institute of Materials Science and Technology, Technische Universität Wien, 1060 Vienna, Austria

adavid.holec@unileoben.ac.at, ${ }^{b}$ paul.mayrhofer@tuwien.ac.at

Keywords: Density functional theory (DFT); Surface energy; Friction coefficient; Multilayer architecture

Abstract. Recently, we have reported on low friction CrN/TiN coatings deposited using a hybrid sputtering technique. These multilayers exhibit friction coefficients $\mu$ below 0.1 when tested in atmosphere with a relative humidity $\approx 25 \%$, but $\mu$ ranges between $0.6-0.8$ upon decreasing the humidity below $5 \%$. Here we use first principle calculations to study $\mathrm{O}$ and $\mathrm{H}$ adatom energetics on TiN and $\mathrm{CrN}$ (001) surfaces. The diffusional barrier of $\mathrm{H}$ on $\mathrm{TiN}(001)$ is about half of the value on $\mathrm{CrN}(001)$ surface, while both elements are stronger bonded on $\mathrm{CrN}$. Based on these results we propose a mechanism for a water-based self-assembled nanobearing.

\section{Introduction}

Chromium and titanium nitride $(\mathrm{CrN}$, TiN) thin films are widely used as hard protective coatings for various industrial and automotive applications as they show high hardness and increased wear and corrosion resistance [1-6]. Tribological investigations of such coatings indicate that the coefficient of friction $\mu$, is around 0.45 and 0.8 for $\mathrm{CrN}$ and TiN coatings, respectively [7-11]. Ehiasarian et al. [12] and Paulitsch et al. [13] showed that using high ionizing deposition techniques such as the high power impulse magnetron sputtering (HIPIMS) for depositing these coatings, leads to increased wear resistance and reduced $\mu$ values due to the formation of dense coating structures [12-15]. Nevertheless, $\mu$ values below 0.1 , comparable to diamond-like carbon coatings or carbo-nitrides, could not be achieved.

Recently, we have deposited multilayer coatings of $\mathrm{CrN}$ and TiN by simultaneously sputter the metal $\mathrm{Cr}$ target in HIPIMS or modulated pulse power (MPP) mode, and the metal $\mathrm{Ti}$ target in direct current magnetron sputtering (DCMS) mode [16, 17]. The resulting films indicate a dense superlattice structure with a bilayer period $\lambda$ from 6 to $10 \mathrm{~nm}$, hardness values $\approx 25 \mathrm{GPa}$, and a preferred (001) orientation [16, 17]. Tribological investigation using a ball-on-disk (BOD) tribometer yielded wear rates $\approx 3 \cdot 10^{-16} \mathrm{~m}^{3} / \mathrm{Nm}$ and a coefficient of friction below 0.1 when tested at room temperature (RT) and relative ambient humidity of around $25 \%$ [16, 17] (see Fig. 1a, curve (1)). Investigations of the triggering effect for the low friction values, by evaluating the wear depth after stopping the BOD testing when the
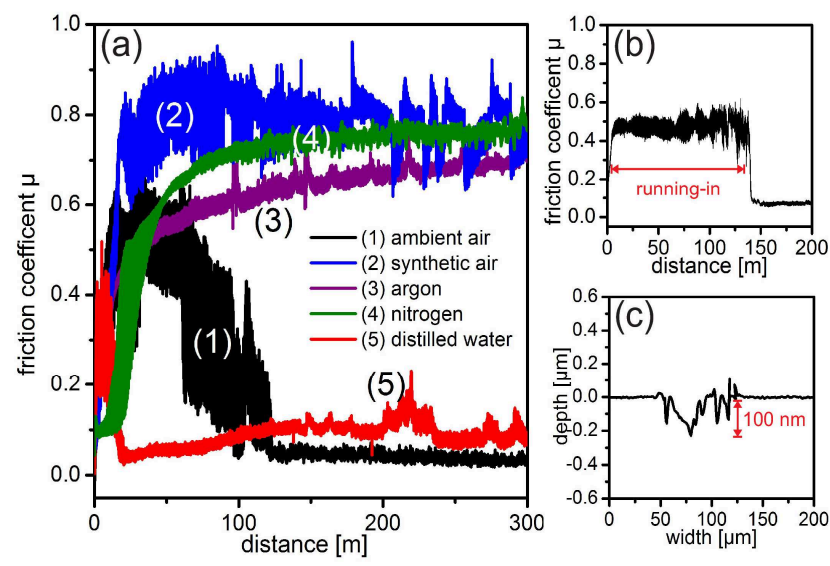

Figure 1: (colour online): (a) BOD tests of $\mathrm{CrN} / \mathrm{TiN}$ superlattice coatings in different atmospheres, published in Ref. [16]. Evaluation of the running-in length, measured in ambient air with a relative humidity of around $25 \%$, and (c) the resulting wear track depth of a $\mathrm{CrN}_{\mathrm{MPP}} / \mathrm{TiN}_{\mathrm{DCMS}}$ multilayer coating with a bilayer period of $10 \mathrm{~nm}$, after stopping the BOD test in the low friction steady state regime. 
$\mu$ value drops below 0.1 , showed that a polishing-in depth of around $100 \mathrm{~nm}$ is necessary, see Figs. $1 \mathrm{~b}$ and c. Furthermore, variations of the ambient air during testing by introducing dry argon, nitrogen or synthetic air, which all reduce the relative humidity to values below $5 \%$, as well as tests in a water bath indicate that the low friction effect of the CrN/TiN multilayer coatings depends sensitively on the relative humidity during testing (see Fig. 1a curves (2) to (5)) [16].

The aim of this study is to clarify the above mentioned observations of water rather than oxygen being the essential ingredient to obtain the low friction coefficient. In this paper we report on density functional theory (DFT) calculation of the hydrogen and oxygen adatom interactions with the free $\mathrm{CrN}$ and $\mathrm{TiN}$ surfaces, as a first approach to the complex interaction between the water molecule and multilayer system.

\section{Methods}

We employed Vienna Ab initio Simulation Package (VASP) $[18,19]$ together with projector augmented wave (PAW) pseudopotentials [20] using the generalised gradient approximation (GGA) as parametrised by Wang and Perdew [21]. The reciprocal space was sampled with minimum of $8000 \mathrm{k}$-points atom and the plane wave cutoff energy was $450 \mathrm{eV}$. The antiferromagnetic configuration of cubic $\mathrm{CrN}$ (B1, $\mathrm{NaCl}$ prototype) was modelled as layers of alternating spins (afm0). Although the true ground state, afm1, has a slightly different arrangement [22-26], the energy of formation, lattice parameters and bulk modulus of these two configurations are very similar (e.g., $\Delta E_{f}^{\text {afm0-afm1 }}=6 \mathrm{meV} /$ at. or $\left.\Delta B_{0}=2 \mathrm{GPa}\right)$. Thus we used the afm0 configuration in all our calculations since it is considerably less computationally demanding than the afml due to a smaller unit cell.

In order to calculate the potential energy surface (PES) for the adatom diffusion on the (001) surfaces, we first optimised the slab and vacuum thicknesses $(\approx 25 \AA$ and $12 \AA$, respectively) for getting converged surface energies. The procedure yielded $60 \mathrm{meV} / \AA^{2}$ for $\mathrm{CrN}(001)$ and $81 \mathrm{meV} / \AA^{2}$ for $\operatorname{TiN}(001)$, the latter value corresponding to those reported in literature $[27,28]$. Subsequently, we used the same slab geometry with an adatom, and for a dense grid of points spanning the (001) surface we optimised the total energy of the system by adjusting the adatom's distance from the surface (with fixed lateral coordinates). The binding energy, $E_{b}$, of an adatom was calculated as

$$
E_{b}=-\left(E_{\text {total }}^{\text {slab+adatom }}-E_{\text {total }}^{\text {slab }}-E_{\text {total }}^{\text {adatom }}\right) .
$$

\section{Results}

Figure 2 shows the PES of oxygen adatom on (001) surface of TiN and $\mathrm{CrN}$. An inspection of the absolute values reveals that $\mathrm{O}$ is stronger bonded to the $\mathrm{CrN}$ surface $\left(E_{b \text {,max }} \approx 5.3 \mathrm{eV}\right)$ than on the TiN surface $\left(E_{b, \max } \approx 4.8 \mathrm{eV}\right)$. Oxygen atoms are strongly bonded in the vicinity of the $\mathrm{Ti}$ and $\mathrm{Cr}$ sites. The $\mathrm{O}$ adatoms are strongly bonded also to the $\mathrm{N}$ sites on the $\mathrm{TiN}(001)$ surface, while the binding is very weak above $\mathrm{N}$ sites on $\mathrm{CrN}(001)$ surface (cf. Figs. 2a and b). The lowest energy barrier (from the PES minimum) for the surface diffusion of $\mathrm{O}$ on the TiN surface is $\approx 0.8 \mathrm{eV}$, corresponding to a movement along the $<110>$ directions, thus suggesting a zig-zag movement between $\mathrm{Ti}$ sites and avoiding $\mathrm{N}$ sites. The lowest diffusion barrier for $\mathrm{O}$ on $\mathrm{CrN}$ is also approximately $0.8 \mathrm{eV}$, however here in the $<100>$ directions. Consequently, oxygen atoms come during the diffusion to the vicinity of both, $\mathrm{Cr}$ and $\mathrm{N}$ atoms. The diffusional behaviour of $\mathrm{O}$ adatoms is therefore qualitatively different on $\mathrm{CrN}$ and $\mathrm{TiN}(001)$ surfaces.

The energetics of $\mathrm{H}$ adatom on TiN and $\mathrm{CrN}$ (001) surfaces is shown in Fig. 3. In contrast to the $\mathrm{O}$ behaviour, hydrogen PES is qualitatively the same for both materials. In both cases, the energetically preferred adatom site is above the $\mathrm{Ti}$ or $\mathrm{Cr}$ atoms, whereas $\mathrm{N}$ sites exhibit local 

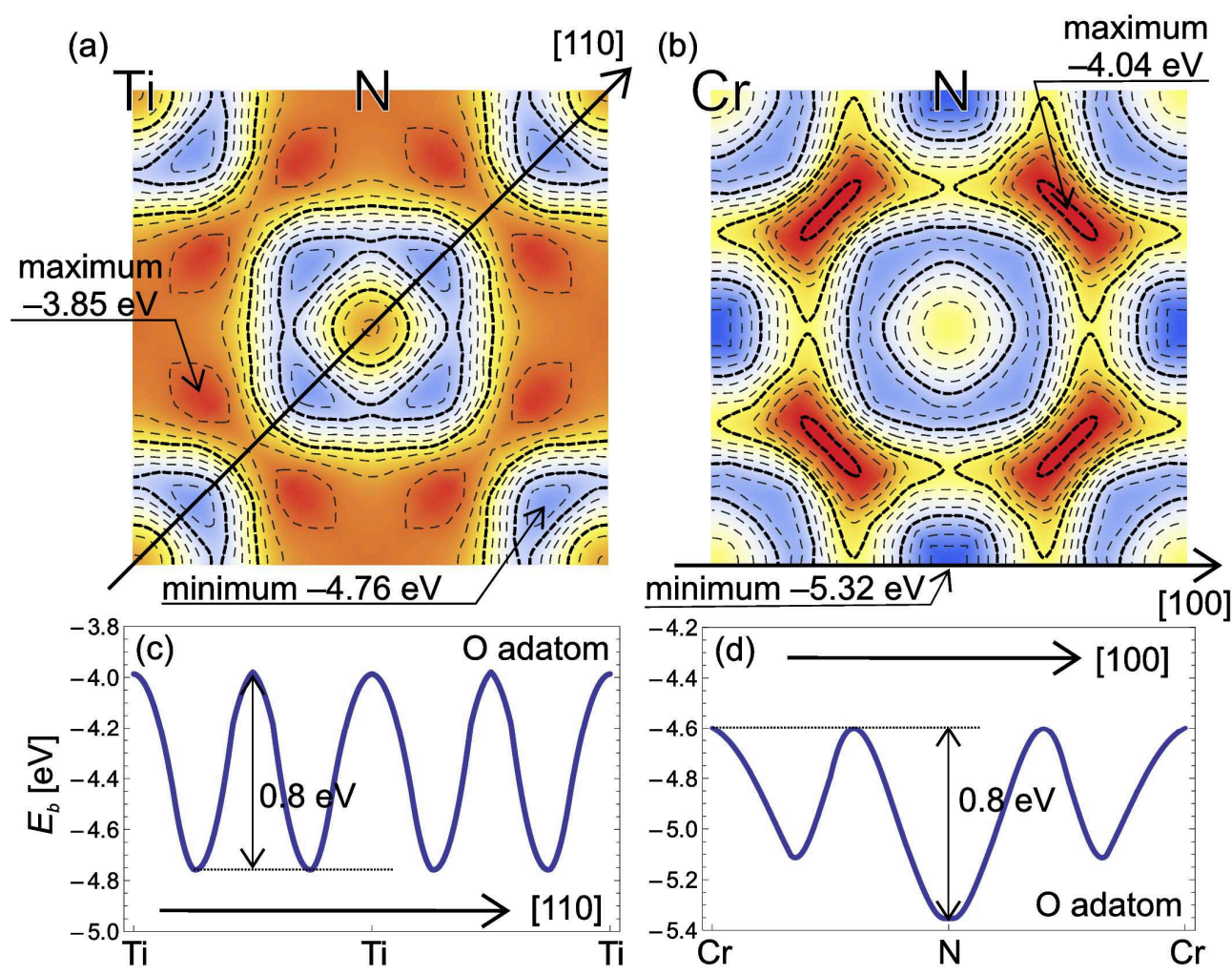

Figure 2: (colour online): Potential energy surface of $\mathrm{O}$ adatom on (001) surface of (a) TiN and (b) $\mathrm{CrN}$. The $2 \mathrm{D}$ cuts in (c) $\mathrm{TiN}<110>$ and (d) $\mathrm{CrN}<100>$ directions show the maximum energy barrier for surface diffusion.

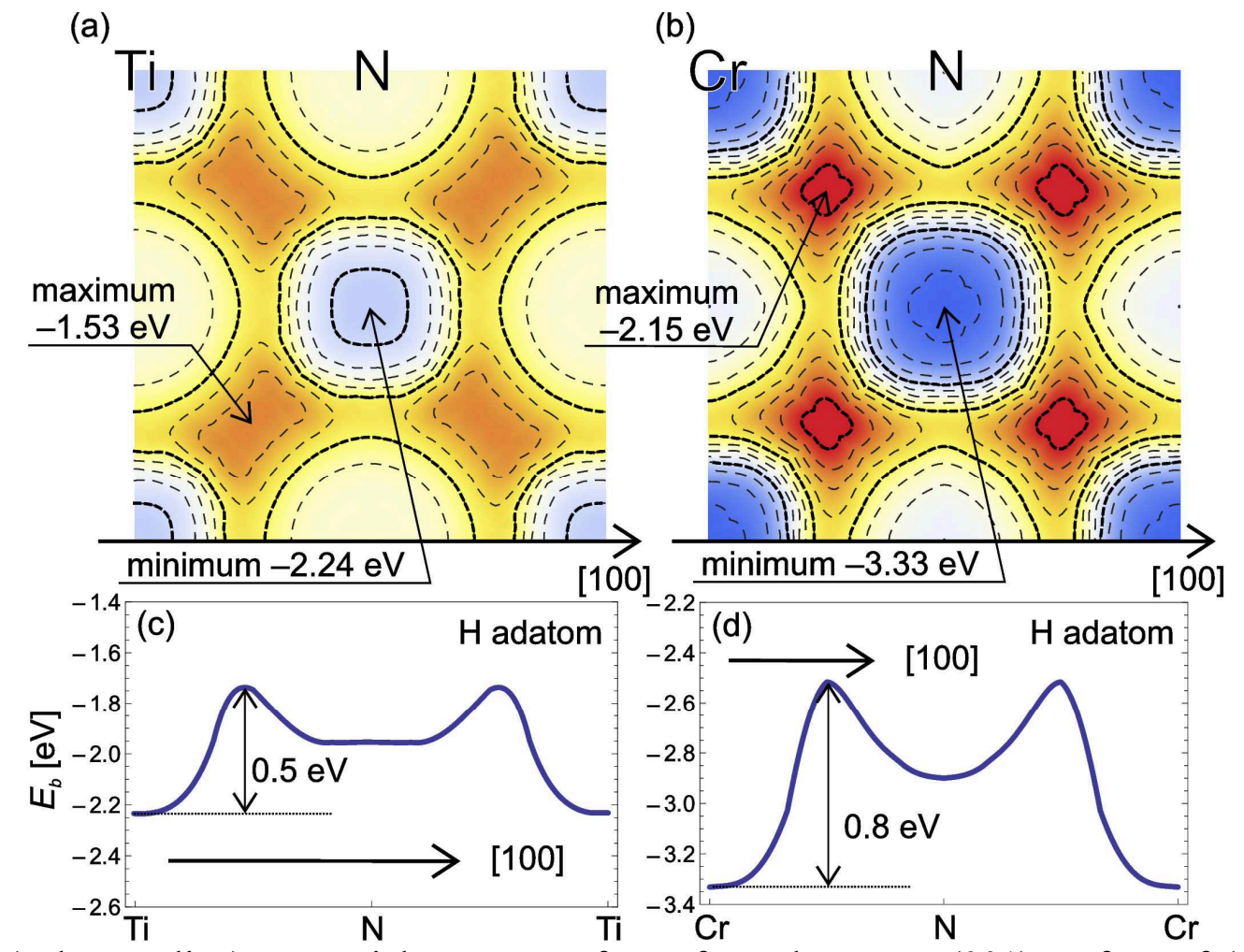

(b)

Figure 3: (colour online): Potential energy surface of $\mathrm{H}$ adatom on (001) surface of (a) TiN and (b) $\mathrm{CrN}$. The $2 \mathrm{D}$ cuts in (c) $\mathrm{TiN}<100>$ and (d) $\mathrm{CrN}<100>$ directions show the maximum energy barrier for surface diffusion. 
minima in PES. The lowest energy barriers for diffusion are along the $<100>$ directions, suggesting that $\mathrm{H}$ atoms come close to both, $\mathrm{Ti}$ or $\mathrm{Cr}$ and $\mathrm{N}$ sites during surface diffusion. Inspection of the PES profiles along the $\langle 100\rangle$ direction, however, reveals that the diffusion barrier is $\approx 0.5$ and $\approx 0.8 \mathrm{eV}$ on $\mathrm{TiN}$ and $\mathrm{CrN}$ surfaces, respectively. As a consequence, $\mathrm{H}$ atoms are predicted to be more mobile on $\operatorname{TiN}(001)$ than on the $\mathrm{CrN}(001)$ surface.

\section{Discussion}

The previous findings may be summarised as follows:

i. $\mathrm{H}$ and $\mathrm{O}$ are stronger bonded on the $\mathrm{CrN}$ than on TiN (001) surface,

ii. the diffusion barriers for $\mathrm{O}$ are comparable on both materials, and

iii. $\mathrm{H}$ diffuses much easier on the TiN surface than on $\mathrm{CrN}$ (the diffusion barrier on the TiN surface is about half of that on $\mathrm{CrN}$ ).

These conclusions are of a direct relevance for the low friction effect of CrN/TiN multilayer coatings provided that water molecules present in the humid atmosphere dissociate and hence act as a source of atomic $\mathrm{O}$ and $\mathrm{H}$ species. In such case, $\mathrm{H}$ (and possibly $\mathrm{O}$, too), would spontaneously pile up on the $\mathrm{CrN}$ while leaving the TiN surface depleted of these species, thus acting as a selfassembled nanobearing (see Fig. 4). Such a mechanism is indirectly supported also by the fact, that in order to get into the low-friction mode, a certain running-in distance is needed first (see Fig. 1). This corresponds to the development of a wear track spanning over several layers (e.g., wear track depth of $\approx 100 \mathrm{~nm}$ for the bi-layer period $\lambda=10 \mathrm{~nm}$; only after a certain number and geometry of layers is exposed to the counterpart surface, the self-assembly of water droplets takes place to promote the low friction. A similar scenario has been used also to explain low friction of Si-doped amorphous carbon coatings when tested in humid atmospheres at elevated temperatures [29].

There is, however, only a limited number of reports on water adsorption on mononitrides. Piscanec et al. [30] used ab initio molecular dynamics to study interaction of a water molecule with TiN(001) surface and concluded that it does not dissociate. Sanyal et al. [31] reached the same conclusion for the TiN(001), however, they predicted a dissociative adsorption on the TiN(110) surface. Our own test calculations (following the procedure in Ref. [31], e.g., static DFT calculations at $0 \mathrm{~K}$ ) of a water molecule on $\mathrm{CrN}(001)$ surface also suggest that the adsorption of a water molecule is not accompanied by its dissociation.

(a)

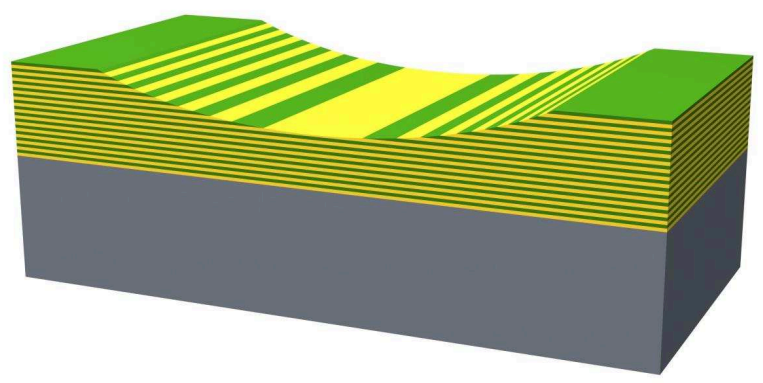

(b)

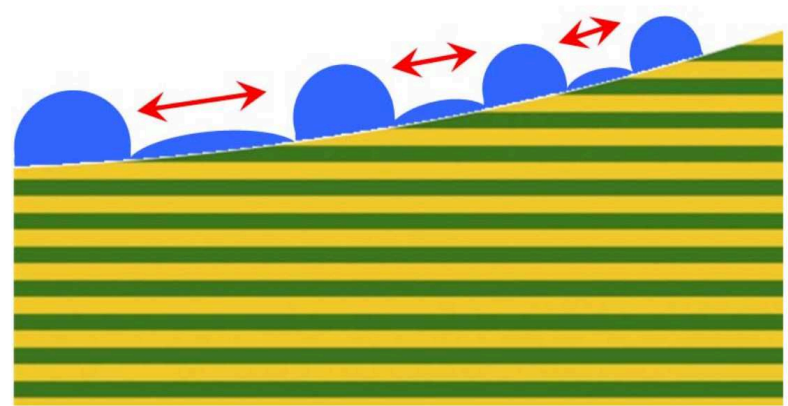

Figure 4: (colour online) A schematic illustration showing the nanobearing effect: (a) a series of alternating TiN (green) and CrN (yellow) layers is obtained after a certain running-in phase, (b) after which the lubricant species adsorbed from the humid atmosphere start preferentially concentrate on $\mathrm{CrN}$ surfaces, hence creating bearing-like effect in the friction contact.

Nevertheless, the real situation is yet much more complicated: due to the polycrystalline nature of sputtered films, there is not a unique surface orientation, moreover, the wear track cuts through the microstructure at different angles, hence exposing different surface orientations (cf. Fig. 4), and finally, the dissociation may be also induced by increased temperature in the wear contact, similarly to what has been reported for a:C [29]. 
It is therefore not conclusive, whether the wear track surface is covered by atomic species or rather by molecules. However, we can also speculate about the behaviour of a water molecule, as an entity bonded either via $\mathrm{O}$ atom or via $\mathrm{H}$ atom to the $\mathrm{CrN} / \mathrm{TiN}$ surface: the water molecule is expected to be more mobile on TiN surface (due to the smaller diffusion barrier for $\mathrm{H}$ atoms) and to be stronger bonded on the $\mathrm{CrN}$. As a consequence of the multilayer (bi-material) arrangement of the $\mathrm{CrN}$ /TiN coatings, water may spontaneously concentrate on the $\mathrm{CrN}$ layers while it depletes on the TiN layers, again leading to the same nanobearing effect.

\section{Conclusions}

In conclusion, we have reported on diffusional properties of $\mathrm{H}$ and $\mathrm{O}$ adatoms on $\mathrm{TiN}$ and $\mathrm{CrN}(001)$ surfaces. $\mathrm{H}$ is shown to be more mobile on TiN, O exhibits the same diffusion barriers on both surfaces. Both elements are stronger bonded on the $\mathrm{CrN}$ than on the TiN surface. Subsequently, we used these results to speculate about the behaviour of water molecules on the CrN/TiN multilayer surface that would rationalise our experimental observations. We propose that the water droplets in the wear track exhibit a tendency for self-assembly with a nanobearing-like effect.

\section{Acknowledgements}

The authors are grateful to the financial support by the START Program (Y371) of the Austrian Science Fund (FWF).

\section{References}

[1] J.-E. Sundgren, Thin Solid Films Vol. 128 (1985), p. 21

[2] J. Musil, S. Kadlec, J. Vyskočil and V. Valvoda, Thin Solid Films Vol. 167 (1988), p. 107

[3] J. Musil, V. Poulek, V. Valvoda, R. Kužel, H. Jehn and M. Baumgärtner, Surf. Coat. Technol. Vol. 60 (1993), p. 484

[4] H. Jensen, U. Jensen, G. Pedersen and G. Sørensen, Surf. Coat. Technol. Vol. 59 (1993), p. 135

[5] T. Hurkmans, D.B. Lewis, J.S. Brooks and W.-D. Münz, Surf. Coat. Technol. Vol. 86-87 (1996), p. 192

[6] T. Hurkmans, Surf. Coat. Technol. Vol. 114 (1999), p 52

[7] S.Y. Lee, G.S. Kim and J.H. Hahn, Surf. Coat. Technol. 177-178, 426 (2004)

[8] E. Badisch, M. Stoiber, G.A. Fontalvo and C. Mitterer, Surf. Coat. Technol. Vol. 174-175 (2003), p. 450

[9] M. Stoiber, E. Badisch, C. Lugmair and C. Mitterer, Surf. Coat. Technol. Vol. 163-164 (2003), p. 451

[10] T. Kacsich, K.P. Lieb, A. Schaper and O. Schulte, J. Phys: Condens. Matter Vol. 8 (1996), p. 10703

[11] J. Takadoum, H. Bennani and M. Allouard, Surf. Coat. Technol. Vol. 88 (1997), p. 232

[12] A.P. Ehiasarian, P.E. Hovsepian, L. Hultman and U. Helmersson, Thin Solid Films Vol. 457 (2004), p. 270

[13] J. Paulitsch, P.H. Mayrhofer, C. Mitterer, W.-D. Münz and M. Schenkel, Proceeding of 50th Annual Technical Conference-Society of Vacuum Coaters (2007), p. 150-154

[14] J. Paulitsch, P.H. Mayrhofer, W.-D. Münz and M. Schenkel, Thin Solid Films Vol. 517 (2008), p. 1239 
[15] J. Paulitsch, M. Schenkel, T. Zufraß, P.H. Mayrhofer and W.-D. Münz, Thin Solid Films Vol. 518 (2010), p. 5558

[16] J. Paulitsch, M. Schenkel, A. Schintlmeister, H. Hutter and P.H. Mayrhofer, Thin Solid Films Vol. 518 (2010), p. 5553

[17] J. Paulitsch, C. Maringer and P.H. Mayrhofer, Tribol. Lett. Vol. 46 (2012), p. 87

[18] G. Kresse and J. Furthmüller, Comput. Mater. Sci. Vol. 6 (1996), p. 15

[19] G. Kresse and J. Hafner, Phys. Rev. B Condens. Matter Vol. 47 (1993), p. 558

[20] G. Kresse and D. Joubert, Phys. Rev. B Condens. Matter Vol. 59 (1999), p. 1758

[21] Y. Wang and J.P. Perdew, Phys. Rev. B Condens. Matter Vol. 44 (1991), p. 13298

[22] L.M. Corliss, N. Elliott and J.M. Hastings, Physical Review Vol. 117 (1960), p. 929

[23] M. Miao and W.R.L. Lambrecht, Phys. Rev. B: Condens. Matter Vol. 71 (2005), p. 1

[24] P.H. Mayrhofer, D. Music, T. Reeswinkel, H.-G. Fuß, and J.M. Schneider, Acta Mater. Vol. 56 (2008), p. 2469

[25] B. Alling, T. Marten, and I.A. Abrikosov, Phys. Rev. B Condens. Matter Vol. 82 (2010), p. 184430

[26] L. Zhou, F. Körmann, D. Holec, M. Bartosik, B. Grabowski, J. Neugebauer, and P.H. Mayrhofer, Phys. Rev. B Condens. Matter Vol. 90 (2014), p. 184102

[27] M. Siodmiak, N. Govind, J. Andzelm, N. Tanpipat, G. Frenking and A. Korkin, Phys. Status Solidi Vol. 226 (2001), p. 29

[28] D. Gall, S. Kodambaka, M.A. Wall, I. Petrov and J.E. Greene, J. Appl. Phys. Vol. 93 (2003), p. 9086

[29] O. Jantschner, S.K. Field, D. Holec, A. Fian, D. Music, J.M. Schneider, K. Zorn and C. Mitterer, Acta Mater. Vol. 82 (2015), p. 437

[30] S. Piscanec, L. Colombi Ciacchi, E. Vesselli, G. Comelli, O. Sbaizero, S. Meriani and A. De Vita, Acta Mater. Vol. 52 (2004), p. 1237.

[31] S. Sanyal, U.V. Waghmare and J.A. Ruud, Appl. Surf. Sci. Vol. 257 (2011), p. 6462 